\title{
The use of technology to provide physical interaction experiences for cognitively able young people who have complex physical disabilities
}

\author{
Mark Moseley \\ Bournemouth University \\ Fern Barrow, \\ Talbot Campus, \\ Poole, Dorset, BH12 5BB \\ mmoseley@bournemouth.ac.uk
}

\begin{abstract}
Young people who have complex physical disabilities and good cognition may face many barriers to learning, communication, personal development, physical interaction and play experiences. Physical interaction and play are known to be important components of child development, but this group currently has few suitable ways in which to participate in these activities.
\end{abstract}

\begin{abstract}
Technology can help to facilitate such experiences. This research aims to develop a technologybased tool to provide this group with the potential for physical interaction and physical play, by providing a means of manipulating objects. The tool will be used to develop the target group's knowledge of spatial concepts and the properties of objects. It will utilise eye gaze technology, robotics and haptic feedback (artificial sensation) in order to simulate physical control and sensations.
\end{abstract}

Throughout this study, there will be a strong focus on Human Computer Interaction, as the target group has complex usability requirements.

Disability, young people, haptic feedback, robotics, eye gaze, $\mathrm{HCl}$, assistive technology.

\section{INTRODUCTION}

Physical disability can prevent young people from having the same real-world experiences as their able-bodied peers. The situation is amplified where there is complex or severe motor impairment, limiting communication and opportunities to interact with the physical world. This, in turn, restricts a person's ability to learn about spatial concepts and, more fundamentally, to engage in physical play. This can hinder the individual's development and constrain participation in activities. It can also result in passivity and a reduced inclination to initiate (Cook et al., 2000). Tactile experiences are frequently facilitated by care-givers or peers using 'hand-over-hand' techniques (Deluca et al., 2006) and, often, physical activities are performed in front of a person while they merely look on (WHO, 2007).

The limitations outlined above impact on development from a young age. Play is regarded as an important component of early child development, especially 'ludic' play or 'play for play's sake' (Missiuna and Pollock, 1991), (Besio et al., 2015) but for those who have physical disabilities, independently initiated physical play can be difficult, or impossible, due to impairments of mobility and dexterity. The situation is exacerbated, as most play materials are not designed with this group's needs in mind (van den Heuvel et al., 2015).

This research aims to provide an ICT-based tool that can provide the potential for the target group (TG) to engage in developing their physical interaction and play skills. The tool will utilise eye gaze technology, robotics and haptic feedback in order to simulate physical control and sensations.

The tool will achieve this goal by allowing the user to control a robotic arm using eye gaze technology. They will be able to instruct the arm to grasp 
objects. When the arm does so, sensors in the 'gripper' will relay sensations back to haptic technology attached to the user's hand, which will then provide a simulated sensation of grasping the object - an action that they would not be able perform themselves.

\section{CONTEXT AND BACKGROUND}

\subsection{Context}

This research is set in the context of human disability. More specifically, it relates to children and young people who have complex physical disabilities which limit their experiences, and to technological advancements that could be used to ameliorate this situation.

\subsection{Background}

The author has worked for two special education schools for the past 10 years, within the fields of ICT and Assistive Technology.

This research focusses on children and young people who present with complex physical disabilities and who would be classified as Level V on the Gross Motor Function Classification System (GMFCS) (Palisano et al., 2007). They will have been assessed as having reached P-Scale (Department for Education, 2014) level 6.0 or above in Mathematics, English and Science.

This group has the cognitive capacity to understand physical concepts but limited opportunities to experience them first-hand. Many physical experiences are currently brought to these children and facilitated by a caregiver.

Children and young people who have complex physical disabilities have fewer opportunities to interact with their environment and partake in activities. Environmental Control Systems (ECS) can provide a degree of control over the physical environment, for example opening doors, turning on lights, interacting with audio-visual equipment and so on, but these lack the haptic sensations of a physical action, which involves intentional touch or grasp.

\section{RESEARCH OBJECTIVES AND QUESTIONS}

This research contains several broad research objectives and research questions:

\subsection{Research Objectives}

- To develop and evaluate touch and haptic approaches appropriate for the TG.

- $\quad$ To create a physical interaction prototype.
- To undertake experiments and evaluation to examine the efficacy of the prototype.

\subsection{Research Questions}

- Can the TG accurately identify physical and haptic feedback sensations?

- Can a prototype be developed to provide the TG with a means to experience physical interaction?

- Does using the prototype improve the TG's understanding of physical concepts?

\section{KEY POINTS OF THE LITERATURE REVIEW}

The importance of physical interaction and play for children with complex disabilities cannot be understated. This group currently has few opportunities in this respect. Overall, few appropriate solutions have been provided, especially regarding ICT toys for play (van den Heuvel et al., 2015).

There are also few interfaces and technologies that allow this group to perform activities such as physical interaction and play.

\subsection{Eye gaze}

Eye gaze is often used for the purpose of monitoring and analysing a person's eyemovements and Point of Regard (POR). However, it can also be used 'interactively' as a means of computer cursor control and selection; in essence, it can replace, or provide a 'hands-free' version of the computer mouse (Sharma and Abrol, 2013), (Duchowski, 2002). This approach affords the user the ability to interact with software-based user interfaces using only their eyes. Eye gaze technology used 'interactively' provides a new direct input access method for the TG (Donegan et al., 2009), allowing them opportunities for communication and environmental control.

Duchowski (2002) broadly categorises approaches to eye gaze interaction techniques, identifying both 'selective' (like a conventional computer mouse, but using, for example, 'dwelling' to 'click') and 'gazecontingent' (adaptive interfaces) (Hansen et al., 2008) methods. Interaction techniques are still emerging, with the most common method used by the TG being 'dwell-clicking'.

Bates et al. (2005) investigated selection techniques using eye gaze within virtual 3D environments, and noted issues including 'loss of context'. The technique described (which involved 'flying' in, or zooming in on an area of interest) would also cause difficulty when attempting to select occluded objects. 


\subsection{Robotics}

Cook and his colleagues have undertaken extensive research involving the use of robotics with children and young people who have disabilities, including robotic manipulators (Cook et al., 2000) and Lego robots (Cook et al., 2012). They state:

\begin{abstract}
"The use of robots may offer a partial solution by providing an opportunity for children to choose how to interact with their environment, to exert some control over the activity and to manipulate three-dimensional objects." (Cook et al., 2012)
\end{abstract}

Some researchers have employed robotics in situations where children who have disabilities can use them in order to achieve an end goal, such as constructing Lego models (Prazak et al., 2004), (Kronreif et al., 2005) or to direct mobile robots within role-play activities (Cook et al., 2012).

Others have used robotics to help this group to interact with their environment (Tsotsos et al., 1998).

\subsection{Haptic feedback}

Example applications of haptic feedback technology use within the context of disability include: rehabilitation 'Wii-hab' (Rebecca The Physio, 2015), boundary definition (Dubey et al., 2010) and tactile sensory feedback (Schwerdt et al., 2009). None of these studies involved the type of children targeted by the current research.

Many existing haptic feedback "gloves" are complex structures that may necessitate long set up periods and require that the user remains very still whilst fitting takes place (Bouzit et al., 2002). Such devices can be restrictive and so are not suited to those who have involuntary movement or contractures, as is the case with the TG. Many of these gloves are prohibitively expensive.

It appears that relatively little is known about using haptic technology to provide the TG with tactile experiences, or indeed, whether it is suitable.

Haptic feedback has been combined with eye gaze technology and some studies have identified it as a useful input modality for reinforcing eye gaze. Käki et al. (2014) demonstrated how haptic feedback can sometimes assist with gaze-controlled tasks. However, Rantala et al. (2014) found that haptic feedback doesn't always assist with gazecontrolled tasks and can sometimes cause confusion or even annoyance. These conflicting results may indicate that further research is needed to identify the most appropriate use cases.

\subsection{Summary}

The literature reports that participants enjoyed using robotics and demonstrated abilities previously unseen, as well as developing new skills (Cook et al., 2005).

Various approaches have combined an input method, for example, switches, with some element of physical control, or have paired an input method with haptic feedback. Other studies have linked eye gaze with haptic feedback. At present, it appears that no one has combined the parts as a whole i.e. eye gaze, robotics and haptic feedback to provide control and simulated 'feel', and then studied the effects upon the TG's development of spatial concepts and knowledge of object properties - the focus of this research study.

In many cases, the existing literature research outputs were not actually used with the intended TG - they were only used with the able-bodied (researchers/students). This research study will be directed by the needs of the participants, who will inform the design and actually use the solutions.

\section{PROBLEM STATEMENT}

Physical interaction and physical play are widely acknowledged to be important factors in child development (Sheridan et al., 1999), (Missiuna and Pollock, 1991), (Besio et al., 2015). The two are intrinsically linked - physical interaction skills are required in order to take part in physical play. However, young people who have complex physical disabilities are largely unable to engage in such situations. This may lead to limited opportunities to participate, initiate, and even to discuss activities, especially if they also have communication difficulties. ICT-based solutions can assist in bridging this gap, but, currently, there are few (van den Heuvel et al., 2015).

\section{RESEARCH APPROACH AND METHODOLOGY}

\subsection{Research Approach}

In this study, the predominant research paradigm, or philosophy being employed is Interpretivism (sometimes described as Phenomenology). This approach is said to be suited to studies involving small samples, where the emphasis is on meaning (Collis and Hussey, 2014).

\subsection{Research Methodology}

This research will use a Mixed Methods or Pragmatic methodology. This approach uses data gathering methods from both the Positivist and Interpretivist paradigms i.e. both quantitative and 
qualitative. The data collection methods used will include surveys, interviews and observation. Pragmatism is said to be 'Problem-Centred' and 'Real-world practice oriented' (Creswell, 2013) which is the basis of this research.

The research sample will be small $(n=5)$ meaning that results will not be statistically significant, and so generalisations will not be meaningful. Instead, the research aims to focus on identifying common themes, and is problem focussed.

\subsection{System Design Method}

The systems design methods employed in this research broadly follow a 'traditional' approach i.e. requirements elicitation, design, implementation, testing and evaluation.

In conventional approaches to systems design, the initial user requirements are often elicited using techniques such as interviews, focus groups and surveys, and by examining any existing systems or procedures. However, this approach is not appropriate for the end users who are the focus of this research project: all of the intended TG have complex physical disabilities and the majority have significant communication difficulties: most are non-verbal, possessing little or no literacy, with their main means of communication being the use of pictorial symbol-based systems combined with eye pointing and eye gaze technology. Whilst these methods work well for basic communication, conveying more complex ideas is challenging, fatiguing and time consuming for this group; they may also lack the expressive language to articulate their thoughts, needs and requirements; added to this is the fact that the problem-space is outside of their experience (they will not have used a system such as this before).

Consequently, rather than the requirements or needs being expressed explicitly by the users, these have been derived from the author's direct experiences and observations of the TG during the past 10 years, whilst working as an Assistive Technologist in special education. This has enabled the author to develop a deeper understanding of this group's abilities and, importantly, what motivates them. Common themes of motivation have emerged including activities involving 'fun', control (especially physical control), creativity (McLoughlin et al., 2016) and those where there is an element of challenge or competition. The control of simple toys and more advanced robotic toys has been found to be very engaging. Preliminary work with an inexpensive robotic arm, led to the inception of this research. Discussion with colleagues (therapy and teaching staff) has suggested that such a tool would be of interest to the TG, and also beneficial from a therapeutic and educational perspective.
As stated previously, the complexity and characteristics of the TG call for flexibility within the design. Prototyping and evolutionary approaches are being adopted in order to develop both the hardware and software components of the tool. This approach allows for an iterative process of continuous refinement.

The TG will be the central focus and involved as much as possible in the development process. However, the TG's limited ability to define their requirements has led to the adoption of a 'proxy' User Centred Design approach i.e. expert users will help to inform the design and evaluate resulting prototypes, on behalf of the TG. This expert user group will comprise some of those who work with the TG, including the author, and therapy and teaching staff employed at the school attended by the TG. These professionals have many years of experience and in-depth knowledge of this group's needs and abilities. Whilst these 'proxy' users will be providing a subjective interpretation of the requirements, which may in fact be very different to those of the TG, their insight will provide useful guidance in the absence of detailed end-user information. The literature also provides insights into designing systems for similar TG's (Cook et al., 2000).

Only once the 'proxy' group have tested and evaluated the resulting prototypes, will the TG be given an opportunity to use them. The TG will be observed using the prototypes and asked to provide feedback, where practicable. Consideration will be given as to the best means for participants to provide this information.

Accessibility is an essential point of focus; each individual has specific needs and abilities and so the user interface and hardware need to be adaptable to suit each person, through customisation and, sometimes, bespoke solutions. An example of this is The Shiva Project (McLoughlin et al., 2016) which identified the importance of a flexible profile-based user interface when creating systems for a range of individuals who have special requirements.

Due to the TG's communication difficulties, it will not be possible for them to describe usability issues or their user experience in detail. For this reason, usability issues and user experience (UX) will be captured using a combination of available feedback and observation. Task directed activities will be undertaken by both 'proxy' and TG participants with results being used to assess system usability and UX. This will help to identify issues and difficulties, which will then inform the development cycle in order to improve the system. 


\section{INITIAL RESULTS}

This research is still in its early stages and so has yet to generate results.

The research has so far produced preliminary working prototypes including:

- A user interface for controlling a robotic arm using eye gaze technology. The interface consists of translucent controls which are overlaid on top of two live webcam feeds onscreen. The user is able to control the arm vertically and horizontally and can open and close the end-effector to grip and release objects. They can also temporarily hide the controls in order to obtain a clearer view from the webcams. The eye gaze unit can be 'paused' when appropriate, giving the user the opportunity to view the scene and onscreen views in order to plan their next moves, but without making selections. Controls are selected by 'dwelling' or gazing at a cell for a predefined period of time.

- A software layer which translates the instructions provided by the user interface into commands which control the movements of a simple robotic arm.

- Electronic circuitry which triggers haptic (vibrating) motors when a pressure sensitive pad is activated. This will form the means of identifying when the robotic arm has gripped an object using its endeffector (gripper/jaws) and relay a sensation to the user's forefinger and thumb.

\section{MAIN CONTRIBUTIONS}

This research is designed to make the following contributions to knowledge:

- Exploring how physical interaction with haptic feedback influences the development of the TG in terms of developing their spatial concepts, knowledge of object properties, and related language. Exploring how haptics can be used to develop body awareness.

- Enabling the TG to control robotics using eye gaze alone.

- Providing the participants with a sensation of performing a physical task.

- Gaining a deeper understanding of how the TG can engage in play-like scenarios and what effect this has upon their development.
- Developing an understanding of the capabilities of this group.

- Providing opportunities for the TG to demonstrate and develop abilities.

Technology outputs:

- Haptic technology appropriate for the TG.

- A suitable interface for selection and manipulation of objects in three dimensions using eye gaze.

- The implementation of an eye gaze controlled robotic arm system with haptic feedback.

To summarise, this research will create a tool involving eye gaze control of robotics in three dimensions, with accompanying haptic feedback. This tool will be used by the TG in order to perform basic physical interaction tasks and physical play activities. This process aims to identify and improve their knowledge of physical spatial concepts and object properties.

\section{REFERENCES}

Bates, R., Istance, H., Donegan, M. \& Oosthuizen, L. (2005). Fly where you look: enhancing gaze based interaction in $3 \mathrm{D}$ environments. Proc. of COGAIN, 5, pp.30-32.

Besio, S., Carnesecchi, M. \& Encarnação, P. (2015). Introducing LUDI: a research network on play for children with disabilities. Studies in Health Technology \& Informatics, 217, 689-695.

Bouzit, M., Burdea, G., Popescu, G. \& Boian, R. (2002). The Rutgers Master II - New design force-feedback glove. IEEE-ASME TRANSACTIONS ON MECHATRONICS, 7, 256263.

Collis, J. \& Hussey, R. (2014). Business research: a practical guide for undergraduate \& postgraduate students, Fourth edition, Basingstoke, Hampshire, Palgrave Macmillan.

Cook, A., Howery, K., Gu, J. \& Meng, M. (2000). Robot enhanced interaction and learning for children with profound physical disabilities. Technology \& Disability, 13, 1-8.

Cook, A. M., Adams, K., Encarnação, P. \& Alvarez, L. (2012). The role of assisted manipulation in cognitive development. Developmental Neurorehabilitation, 15, 136-148.

Cook, A. M., Bentz, B., Harbottle, N., Lynch, C. \& Miller, B. (2005). School-based use of a robotic arm system by children with disabilities. Neural 
Systems and Rehabilitation Engineering, IEEE Transactions on, 13, 452-460.

Creswell, J. W. (2013). Research design : qualitative, quantitative, and mixed method approaches, Los Angeles, Calif. : SAGE, 2013. Fourth edition, international student edition.

Deluca, S. C., Echols, K., Law, C. R. \& Ramey, S. L. (2006). Intensive Pediatric Constraint-Induced Therapy for Children With Cerebral Palsy: Randomized, Controlled, Crossover Trial. Journal of Child Neurology, 21, 931-938.

Department for Education. (2014). Performance - $P$ Scale - attainment targets for pupils with special educational needs. Available from https://www.gov.uk/government/uploads/system/ uploads/attachment data/file/329911/Performan ce - P Scale -

attainment targets for pupils with special ed ucational needs.pdf (14/06/2016).

Donegan, M., Morris, J. D., Corno, F., Signorile, I., Chió, A., Pasian, V., Vignola, A., Buchholz, M. \& Holmqvist, E. (2009). Understanding users and their needs. Universal Access in the Information Society, 8, 259-275.

Dubey, V. N., Klopot, W. \& Skupin, P. (2010). Implementation of virtual control strategies for natural rehabilitation of arm with visual and force feedback. 2010 IEEE International Conference on Robotics \& Biomimetics (ROBIO), 69.

Duchowski, A. T. (2002). A breadth-first survey of eye-tracking applications. Behavior Research Methods, Instruments \& Computers, 34, 455470.

Hansen, D.W., Skovsgaard, H.H., Hansen, J.P. And Møllenbach, E. (2008), March. Noise tolerant selection by gaze-controlled pan and zoom in 3D. In: Proceedings of the 2008 symposium on Eye tracking research \& applications. 205-212.

Kronreif, G., Prazak, B., Mina, S., Kornfeld, M., Meindl, M. And Fürst, M. (2005). Playrob-robotassisted playing for children with severe physical disabilities. In: Rehabilitation Robotics, 2005. ICORR 2005. 9th International Conference on 193-196.

Käki, K., Majaranta, P., Špakov, O. And Kangas, J., (2014), October. Effects of haptic feedback on gaze based auto scrolling. In: Proceedings of the 8th Nordic Conference on Human-Computer Interaction: Fun, Fast, Foundational. 947-950.

McLoughlin, L., Fryazinov, O., Moseley, M., Sanchez, M., Adzhiev, V., Comninos, P. \& Pasko, A. (2016). Virtual Sculpting and 3D Printing for Young People with Disabilities. IEEE Computer Graphics \& Applications, 36, 22-28.
Missiuna, C. \& Pollock, N. (1991). Play Deprivation in Children With Physical Disabilities: The Role of the Occupational Therapist in Preventing Secondary Disability. American Journal of Occupational Therapy, 45, 882-888.

Palisano, R., Rosenbaum, P., Bartlett, D. \& Livingston, M. (2007). Gross Motor Function Classification System - Expanded and Revised (GMFCS - E \& R) - Level V. 2015. Available from

https://canchild.ca/system/tenon/assets/attachm ents/000/001/399/original/GMFCS English Illust rations.pdf (14/06/2016).

Prazak, B., Kronreif, G., Hochgatterer, A. \& Fürst, M. (2004). A toy robot for physically disabled children. Technology \& Disability, 16, 131-136.

Rantala, J., Kangas, J., Akkil, D., Isokoski, P. \& Raisamo, R. (2014). Glasses with haptic feedback of gaze gestures. Conference on Human Factors in Computing Systems Proceedings, 1597.

Rebecca The Physio. (2015). wiihabilitation. Available from http://www.wiihabilitation.co.uk/. (14/06/2016).

Schwerdt, H.N., Tapson, J. and EtienneCummings, R. (2009), March. A color detection glove with haptic feedback for the visually disabled. In: Information Sciences and Systems, 2009. CISS 2009. 43rd Annual Conference on. 681-686.

Sharma, A. \& Abrol, P. (2013). Eye Gaze Techniques for Human Computer Interaction: A Research Survey. International Journal of Computer Applications, 71.

Sheridan, M. D., Harding, J. \& Meldon-Smith, L. (1999). Play in early childhood : from birth to six years, London : Routledge, 1999. [2nd ed] / revised and updated by Jackie Harding and Liz Meldon-Smith.

Tsotsos, J. K., Verghese, G., Jepson, A., Nuflo, F., Culhane, S., Dickinson, S., Stevenson, S., Jenkin, M., Milios, E., Black, M., Metaxas, D., Ye, Y. \& Mann, R. (1998) PLAYBOT: A visuallyguided robot for physically disabled children. Image and Vision Computing, 16, 275-292.

Van Den Heuvel, R., Lexis, M. \& De Witte, L. (2015) ICT based technology to support play for children with severe physical disabilities. Studies in Health Technology \& Informatics, 217, 573577.

WHO. (2007). International classification of functioning, disability and health: children and youth version: ICF-CY, Geneva, World Health Organization. 